\title{
Time-Domain Studies of Gravitationally Lensed Quasars
}

\author{
Luis J. Goicoechea ${ }^{1}$ and Vyacheslav N. Shalyapin ${ }^{1,2} \dagger$ \\ ${ }^{1}$ Dept. de Física Moderna, Universidad de Cantabria, ES-39005, Santander, Spain \\ email: goicol@unican.es \\ ${ }^{2}$ Inst. Radiophys. \& Elect., Nat. Acad. Sci. Ukraine, UA-61085, Kharkov, Ukraine \\ email: vshal@ukr.net
}

\begin{abstract}
We present an overview and current results of an ongoing optical/NIR monitoring of seven gravitationally-lensed quasars (GLQs) with the 2-m Liverpool Robotic Telescope. The photometric data from the first seven years (2005-2011) of this programme are leading to high-quality light curves, which in turn are being used as key tools for different standard and novel studies. While brightness records of non-lensed distant quasars may contain unrecognized extrinsic variations, one can disentangle intrinsic from extrinsic signals in certain GLQs. Thus, some GLQs in our sample allow us to assess their extrinsic and intrinsic variations; we then discuss the origin of both kinds of fluctuations. We also demonstrate the usefulness of GLQ time-domain data for obtaining successful reverberation maps of the inner regions of accretion disks around distant supermassive black holes, and for estimating the redshifts of distant lensing galaxies.
\end{abstract}

Keywords. gravitational lensing, black hole physics, accretion, galaxies: general, quasars: general.

An overview of our ongoing Liverpool Quasar Lens Monitoring (LQLM) project is presented in Table 1. The data collection are being carried out in different phases: LQLM I (from 2005 January to 2007 July), LQLM II (from 2008 February to 2010 July) and LQLM III (from 2010 October to the present), and is using available optical/NIR instrumentation. The relevant instruments on the Liverpool Robotic Telescope are the RATCam CCD camera and its associated Sloan griz filter set, the RINGO2 optical polarimeter, and the FRODOspec spectrograph (3900-9400 A). Some astrophysical results and expectations for each target GLQ are given here; a more complete and updated information can be found on the GLENDAMA website http://grupos.unican.es/glendama.

SBS 0909+532. The LQLM I light curves in the $r$ band led to a robust time-delay between its two images of $\Delta t_{A B}=-49 \pm 6$ days and $\Delta t_{i j}=t_{j}-t_{i}$, B leading (Goicoechea et al. 2008a). In addition, the optical flux ratio $A / B$ changed little in the first 10 years of observations, i.e., between the identification as a quasar pair in 1996 and our LQLM I campaign (see Dai \& Kochanek 2009 and references therein). For example, the $r$-band light curve of the A image and the properly shifted $r$-band light curve of $\mathrm{B}$ were consistent with each other throughout the LQLM I period, so the variability over this time segment was basically intrinsic to the distant quasar (Goicoechea et al. 2008a). However, the LQLM III light curves indicate that the $r$-band flux ratio had evolved in 2010-2011. Gravitational microlensing by stars within the main lensing galaxy could account for the detected extrinsic variation.

FBQ 0951+2635. Gravitational microlensing seems to be an important variability mechanism for this GLQ (Paraficz et al. 2006; Shalyapin et al. 2009). We are taking a few frames per year in the $r$ band to trace the long-term behaviour of $A / B$, and thus to

$\dagger$ On behalf of the GLENDAMA Project Team. 
Table 1. Current status of the LQLM project.

\begin{tabular}{|c|c|c|c|c|c|}
\hline $\begin{array}{l}\text { GLQ } \\
\text { (redshift) }\end{array}$ & Comments $^{1}$ & $\begin{array}{l}\text { Main lens } \\
\text { (redshift) }\end{array}$ & $\begin{array}{c}\text { Observation } \\
\text { phases }^{2}\end{array}$ & Instruments ${ }^{2}$ & $\begin{array}{l}\text { Outputs }^{3} \\
\text { (status) }\end{array}$ \\
\hline $\begin{array}{l}\text { SBS } 0909+532 \\
(z=1.38)\end{array}$ & \begin{tabular}{|l}
2 images: $\mathrm{A}-\mathrm{B}$ \\
size $\sim 1.11^{\prime \prime}$
\end{tabular} & \begin{tabular}{|c} 
early-type galaxy \\
$(z=0.83)$
\end{tabular} & $I+U I$ & $\begin{array}{l}\text { RATCam } \\
g r \text { filters }\end{array}$ & $\begin{array}{c}\mathrm{LC} \\
\text { (final reduction) }\end{array}$ \\
\hline $\begin{array}{l}\text { FBQ } 0951+2635 \\
(z=1.25)\end{array}$ & $\left|\begin{array}{c}2 \text { images: } \mathrm{A}-\mathrm{B} \\
\text { size } \sim 1.10^{\prime \prime}\end{array}\right|$ & \begin{tabular}{|c} 
early-type galaxy \\
$(z=0.26)$
\end{tabular} & $\mathrm{I}+\mathrm{II}+\mathrm{III}$ & $\begin{array}{l}\text { RATCam } \\
\text { ri filters }\end{array}$ & $\begin{array}{c}\mathrm{LC}+\mathrm{DI} \\
\text { (final reduction) }\end{array}$ \\
\hline $\begin{array}{l}\text { QSO } 0957+561 \\
(z=1.41)\end{array}$ & $\mid \begin{array}{c}2 \text { images: } \mathrm{A}-\mathrm{B} \\
\text { size } \sim 6.17^{\prime \prime}\end{array}$ & $\begin{array}{l}\mathrm{cD} \text { galaxy } \\
(z=0.36)\end{array}$ & $\mathrm{I}+\mathrm{II}+\mathrm{III}$ & $\begin{array}{l}\text { RATCam } \\
\text { griz filters } \\
\text { FRODOspec } \\
\text { RINGO2 }\end{array}$ & $\begin{array}{c}\mathrm{LC}+\mathrm{S}+\mathrm{PM} \\
(\mathrm{LC}=\text { final reduction, } \\
\mathrm{S}=\text { first reduction, } \\
\mathrm{PM}=\text { pending })\end{array}$ \\
\hline $\begin{array}{l}\text { SDSS } 1001+5027 \\
(z=1.84)\end{array}$ & $\left|\begin{array}{c}2 \text { images: A-B } \\
\text { size } \sim 2.86^{\prime \prime}\end{array}\right|$ & \begin{tabular}{|c} 
early-type galaxy \\
$(z \sim 0.2-0.5)$
\end{tabular} & II & $\begin{array}{l}\text { RATCam } \\
g \text { filter }\end{array}$ & $\begin{array}{c}\mathrm{LC} \\
\text { (final reduction) }\end{array}$ \\
\hline $\begin{array}{l}\text { SDSS } 1339+1310 \\
(z=2.24)\end{array}$ & $\left|\begin{array}{c}2 \text { images: A-B } \\
\text { size } \sim 1.69^{\prime \prime}\end{array}\right|$ & $\begin{array}{c}\text { early-type galaxy } \\
(z \sim 0.4)\end{array}$ & $\mathrm{II}+\mathrm{III}$ & $\begin{array}{l}\text { RATCam } \\
r i \text { filter }\end{array}$ & $\begin{array}{c}\mathrm{LC}+\mathrm{DI} \\
\text { (first reduction) }\end{array}$ \\
\hline $\begin{array}{l}\mathrm{HE} 1413+117 \\
(z=2.56)\end{array}$ & $\left|\begin{array}{c}4 \text { images: A-D } \\
\text { size } \sim 1.35^{\prime \prime}\end{array}\right|$ & $\begin{array}{c}? \\
(z=1.9)\end{array}$ & II & $\begin{array}{l}\text { RATCam } \\
r \text { filter }\end{array}$ & $\begin{array}{c}\mathrm{LC}+\mathrm{DI} \\
\text { (final reduction) }\end{array}$ \\
\hline $\begin{array}{l}\text { QSO } 2237+0305 \\
(z=1.69)\end{array}$ & $\left|\begin{array}{c}4 \text { images: A-D } \\
\text { size } \sim 1.78^{\prime \prime}\end{array}\right|$ & \begin{tabular}{|} 
face-on Sb galaxy \\
$(z=0.04)$
\end{tabular} & II & $\begin{array}{l}\text { RATCam } \\
g r \text { filters }\end{array}$ & $\begin{array}{c}\text { LC } \\
\text { (first reduction) }\end{array}$ \\
\hline
\end{tabular}

Notes:

${ }^{1}$ See the CASTLES (http://www.cfa.harvard.edu/castles/) and

SQLS (http://www-utap.phys.s.u-tokyo.ac.jp/ sdss/sqls/) websites.

${ }^{2}$ See main text.

${ }^{3} \mathrm{LC}=$ light curves, $\mathrm{DI}=$ deep images, $\mathrm{S}=$ spectra, $\mathrm{PM}=$ polarization measurements.

obtain information about the structure of the source and the lensing galaxy (Wambsganss 1990; Kochanek 2004).

QSO $0957+561$. We did not find evidence of extrinsic variability in the LQLM I light curves in the $g$ and $r$ bands. These initial brightness records were used to measure time delays between images and optical bands (Shalyapin et al. 2008), and to analyse the structure function of the rest-frame UV variability (Goicoechea et al. 2008b; Goicoechea et al. 2010). Later, LQLM II fluxes in the griz bands, together with concurrent spacebased observations from SWIFT/UVOT and Chandra, unveiled details of the accretion flow and its jet connection in a distant radio-loud quasar for the first time (see Gil-Merino et al. 2011). Our global database in the $g r$ bands is also providing surprising results on the chromaticity in $\Delta t_{A B}$ and the long-term evolution of $B / A$, which are probably related to the presence of a dense cloud within the $\mathrm{cD}$ lensing galaxy along the line of sight to the A image. We are also exploring the spectro-polarimetric evolution of this fascinating first GLQ.

Two new GLQs. SDSS 1001+5027 was discovered in 2005 (Oguri et al. 2005). The first monitoring campaign in the $R$ band did not produce any time delay between its two images (Paraficz et al. 2009). We have recently observed this double GLQ in the $g$ band (February-May 2010), since we expect to see more variability at shorter wavelengths. If A leads B, and there are no significant extrinsic variations, the LQLM II $g$-band fluxes suggest a time delay ranging from 12 to 22 days. The other new GLQ (SDSS 1339+1310; Inada et al. 2009) was monitored in the $r$ band just after its discovery (February-July 2009; LQLM II). Although Fig. 1 shows prominent flux variations, our LQLM II $r$-band light curves do not reveal any conclusive delay. Additional data during LQLM III are required in order to decide on the time delay and other properties of this system.

Two famous quads. We followed up the $r$-band variability of the four images A-D of the Cloverleaf quasar (HE 1413+117) in order to measure its time delays for the first time. The LQLM II fluxes of this GLQ (February-July 2008) enabled us to obtain 


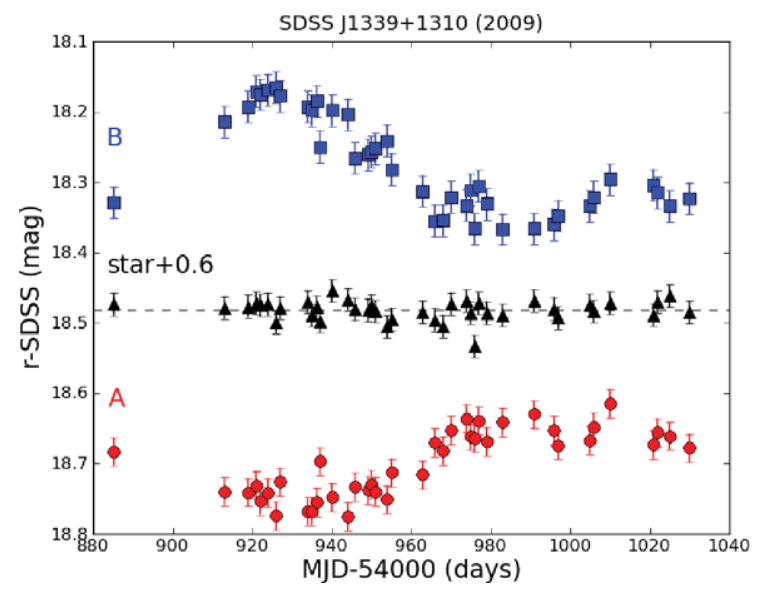

Figure 1. LQLM II $r$-band light curves of SDSS $1339+1310$.

$\Delta t_{A B}=-17 \pm 3$ days, $\Delta t_{A C}=-20 \pm 4$ days and $\Delta t_{A D}=23 \pm 4$ days (B-C leading, $\mathrm{D}$ trailing), which we then used to estimate the redshift of the main lensing galaxies: $z=$ $1.88_{-0.11}^{+0.09}$ (Goicoechea \& Shalyapin 2010). Although useful spectroscopic data are not yet available, we derived an accurate value of $z$ via gravitational lensing. We also monitored the Einstein Cross (QSO 2237+0305) in the $g$ and $r$ bands; the light curves and microlensing analyses will be presented soon. QSO $2237+0305$ is the most emblematic target for microlensing studies (Shalyapin et al. 2002; Kochanek 2004; Gil-Merino et al. 2006).

\section{References}

Dai, X. \& Kochanek, C. S. 2009, ApJ, 692, 677

Gil-Merino, R., González-Cadelo, J., Goicoechea, L. J., Shalyapin, V. N., \& Lewis, G. F. 2006, MNRAS, 371, 1478

Gil-Merino, R., Goicoechea, L. J., Shalyapin, V. N., \& Braga, V. F. 2011, ApJ, in press (arXiv:1109.3330)

Goicoechea, L. J. et al. 2008a, New Astron., 13, 182

Goicoechea, L. J., Shalyapin, V. N., Gil-Merino, R., \& Ullán, A. 2008b, A\&\&A, 492, 411

Goicoechea, L. J., Shalyapin, V. N., \& Gil-Merino, R. 2010, The Open Astron. J., 3, 193 (see http://www. benthamscience.com/open/toaaj/)

Goicoechea, L. J. \& Shalyapin, V. N. 2010, ApJ, 708, 995

Inada, N., et al. 2009, $A J, 137,4118$

Kochanek, C. S., 2004, ApJ, 605, 58

Oguri, M., et al. 2005, ApJ, 622, 106

Paraficz, D., Hjorth, J., Burud, I., Jakobsson, P., \& Elíasdóttir, Á. 2006, A\&̧A, 455, L1

Paraficz, D., Hjorth, J., \& Elíasdóttir, Á. 2009, A\&A A, 499, 395

Shalyapin, V. N. et al. 2002, ApJ, 579, 127

Shalyapin, V. N., Goicoechea, L. J., Koptelova, E., Ullán, A., \& Gil-Merino, R. 2008, A\&A A, 492, 401

Shalyapin, V. N., et al. 2009, MNRAS, 397, 1982

Wambsganss, J., 1990, PhD thesis, Munich University (also available as report MPA 550) 